Revue internationale P.M.E.

Économie et gestion de la petite et moyenne entreprise

Revue

internationale

PME

\title{
La mercatique de la petite entreprise
}

\section{Michel Marchesnay}

Volume 1, numéro 3-4, 1988

URI : https://id.erudit.org/iderudit/1007884ar

DOI : https://doi.org/10.7202/1007884ar

Aller au sommaire du numéro

Éditeur(s)

Presses de l’Université du Québec

ISSN

0776-5436 (imprimé)

1918-9699 (numérique)

Découvrir la revue

Citer cet article

Marchesnay, M. (1988). La mercatique de la petite entreprise. Revue internationale P.M.E., 1(3-4), 259-276. https://doi.org/10.7202/1007884ar

\section{Résumé de l'article}

Les petites et toutes petites entreprises utilisent des méthodes spécifiques pour étudier leur marché et développer une action commerciale. La spécificité est due à la nature de l'entrepreneur, aux conditions de l'environnement

(vulnérabilité, dépendance, agressivité), au choix des produits et des marchés. Il apparaît principalement que la petite entreprise a une activité commerciale fondée sur les relations personnelles des dirigeants avec les clients. Le plan de marchéage est inexistant. L'article s'inspire de l'observation de 37 entreprises de 3 régions différentes de France. 


\title{
La mercatique de la petite entreprise
}

\author{
Michel MARCHESNAY \\ ERFI, Université de Montpellier
}

\begin{abstract}
RÉSUME
Les petites et toutes petites entreprises utilisent des méthodes spécifiques pour étudier leur marché et développer une action commerciale. La spécificité est due à la nature del'entrepreneur, aux conditions de l'environnement (vulnérabilité, dépendance, agressivité), au choix des produits et des marchés. II apparaît principalement que la petite entreprise a une activité commerciale fondée sur les relations personnelles des dirigeants avec les clients. Le plan de marchéage est inexistant. L'article s'inspire de l'observation de 37 entreprises de 3 régions différentes de France.
\end{abstract}

\begin{abstract}
The small and smaller business firms are using specific methods for market research and market development. The specificity is linked with the nature of the entrepreneur, with the environmental features (vulnerability, dependance, agressivity), with the choice of products and markets. It mainly appears that the SBF has a trade activity based upon the personnal relations of the manager with the customers. The use of marketing mix is unexistant. This paper is inspired by the observation of 37 SBF in 3 different French areas.
\end{abstract}

\section{RESUMEN}

Las pequeñas y muy pequeñas empresas emplean metódos specificos para estudiar su mercado y desarrollar una acción comercial. La specificidad es causada por la natura del empresario, las condiciones del entorno (vulnerabilidad, dependencia, agresividad) y las elecciones de productos y de mercados. Aparece principalmente que la pequeña empresa tiene una actividad comercial centrada sobre las relaciones personales del dirigente con los clientes. No existe el «marketing mix». El artículo es inspirado por la observación de 37 pequeñas empresas de 3 diferentes regiones de Francia.

- Docteur en sciences économigues, professeur en sciences de gestion à l'Université de Montpellier 1 et directeur de l'Equipe de recherche sur la firme et l'industrie, le professeur Marchesnay a publié de nombreux ouvrages et articles sur les P.M.E. ainsi que des rapports destinés aux instances gouvernementales sur cette question (adresse: ERFI, Université de Montpellier, 14, rue du Cardinal de Cabrières, $4^{4}$ étage, 34060 Montpellier Cedex, France). Cet article reçu en avril 1989 est une version reprise d'une communication présentée au colloque de marketing de Bilbao, Espagne. 
La mercatique, ou pour reprendre un vocable davantage usité le marketing, a fait l'objet de rares investigations dans son application à la petite entreprise. Comme le souligne J. Kinsey (Kinsey, 1987), «très peu de choses ont été écrites sur l'emploi par les petites entreprises du "marketing mix"». Rares sont, dans les manuels de marketing ou de gestion commerciale, les allusions à la situation particulière qui lui soient spécifiquement consacrées ${ }^{1}$.

Or, il s'agitlàautant d'un champde recherches qued'un lieu de préoccupations particulièrement sensible. La petite entreprise, qui occupe, pour faire bref, moins de cinquante salariés, a connu, au cours de cette dernière décennie, une ascension fulgurante (Julien et Marchesnay, 1988). Cette ascension repose davantage sur la hausse de la fécondité que sur la baisse de la mortalité : l'extrême vulnérabilité de la petite entreprise vis-à-vis de son environnement de marché la prédispose à subir des attaques souvent mortelles, au cours des premières années d'existence; et l'absence d'une analyse systématique du marché empêche toute immunisation. Parmi les causes de mortalité et de défaillance, l'absence d'une politique mercatique est des plus fréquemment citée, consécutivement à une appellation générique de «mauvaise gestion».

Toutefois, il convient de ne pas tomber dans les facilités d'une vision trop normative, dans laquelle la faute incomberait totalement aux propriétaires-dirigeants de petites entreprises, accusés de ne pas se plier aux préceptes de la mercatique. Il semble bien que ces préceptes, tels qu'ils sont couramment enseignés et proposés par les cabinets de conseil en marketing, soient fortement déconnectés des besoins et des pratiques de la petite entreprise. Un large effort d'adaptation reste donc à faire.

Aussi convenait-il d'observer les pratiques commerciales des dirigeants de petite entreprise, au travers d'enquêtes directes, afin de constater si, effectivement, une certaine propension à éluder les problèmes commerciaux et de marché pouvait se vérifier peu ou prou. Une telle recherche, répétons-le, n'est pas si fréquente : on retiendra, notamment, l'étude de J. Kinsey, précédemment citée.

Préalablement à toute observation de terrain, il importe de préciser les traits distinctifs de la petite et toute petite entreprise, et de se demander, a priori, quels effets peuvent entraîner ceux-ci sur l'activité commerciale du dirigeant-propriétaire. A ce stade de la réflexion, il faut tenter de privilégier des variables et des facteurs explicatifs de ces comportements particuliers.

Dans une seconde étape, il convient de se pencher sur la réalité concrète, au travers d'entretiens directs avec des dirigeants. Cette solution est nettement supérieure à l'enquête par questionnaire postal, surtoutàce stade qui ne peutêtre qu'exploratoire, face à un problème non inventorié. Par ailleurs, il ne saurait être question de se fixer

1 L'ouvrage de Bertrand Saporta (Saporta, 1986), aborde le problème de la stratégie commerciale des moyennes entreprises, c'est-à-dire d'entreprises structurées de manière à disposer d'une organisation commerciale. Tel n'est pas le cas des petites entreprises que nous étudions. 
comme objectif le choix d'un échantillon statistiquement significatif, toujours à ce stade de la recherche. En effet la petite entreprise se caractérise par une très grande diversité dans les structures comme dans les problèmes. Il semble donc préférable, à la réflexion, de comparer de petits échantillons dans des populations différentes, mais qui nous apparaissent bénéficier chacune d'une relative homogénéité interne, ce qui devrait permettre de dégager des différences et des ressemblances propres à susciter des hypothèses de travail sur le sens des relations entre variables supposées pertinentes.

Celles-ci seront abordées sous trois thèmes:l'entrepreneur, l'environnement, l'activité. Répétons qu'à ce stade de la recherche, les propos que nous tiendrons relèvent autant d'indications normatives que d'observations empiriques, étayées de surcroît par notre propre expérience de terrain, et confrontées à la (rare) littérature sur la question.

\section{$1 \quad$ Spécificité de la petite entreprise}

Les auteurs spécialistes de la petite entreprise ne s'en tiennent généralement pas à une simple définition en termes d'effectifs (par exemple : moins de cinquante salariés), mais s'attachent au contraire à préciser les traits distinctifs de ce type d'organisation (Naro, 1989; Julien et Marchesnay, 1988).

Les traits les plus généralement retenus sont les suivants :

\subsection{Une direction très personnalisée}

Le personnage central de la petite entreprise est le propriétaire-dirigeant.

- Le propriétaire-dirigeant est l'entrepreneur : il est à la base de la création de l'entreprise et de l' «innovation», c'est-à-dire de l'idée qui justifie cette création, idée plus ou moins neuve au demeurant. Les enquêtes relatives aux raisons de la création privilégient les causes suivantes :

- exploiter une idée technique (nouveau procédé ou savoir-faire)

- exploiter une idée commerciale (nouveau marché, nouveau produit, nouveau besoin);

- réaliser une aspiration personnelle : indépendance, enrichissement, exploitation de l'expérience acquise à des fins personnelles, travailler en communauté, créer quelque chose. Bref, un ensemble d'aspirations égoïstes et altruistes, pas toujours bien discernées.

Il apparaît que ces mobiles exercent une influence sans doute non négligeable sur l'intérêt manifesté à l'égard des activités liées au marché. Ainsi, s'il s'agit d'exploiter une idée de marché, on peut penser que l'étude du marché sera plus 
approfondie. Inversement, l'idée d'exploiter une technique peut conduire à sousévaluer l'importance de l'action commerciale.

- Le propriétaire-dirigeant est le manageur. A ce titre, il dispose d'un certain nombre de compétences directoriales. Celles-ci reposent sur les critères suivants :

- le niveau de formation : études supérieures ou non;

- le type de formation : études techniques, de gestion (notamment commerciales), autres;

- le degré d'expérience antérieure : aucune, expérience comme exécutant, cadre ou dirigeant.

On peut penser que, plus le dirigeant est formé en gestion à un niveau supérieur, plus il a d'expérience de direction, plus il manifestera de propension à s'occuper des problèmes de marché.

- Le propriétaire-dirigeant est l'organisateur. Dans la petite entreprise, et plus celle-ci est petite, l'organisation tourne autour de son dirigeant, lequel constitue la plaque tournante du système d'information et de décision. Le pouvoir est donc extrêmement personnalisé, et l'analyse du temps passé dans les diverses activités par le dirigeant est, du point de vue de la recherche, tout à fait éclairant (Mintzberg, 1984, Fallery, 1983).

Il convient de distinguer la nature des activités :

- activités de contrôle ou de décision, voire d'opération;

- activités générales ou sur une fonction donnée.

A priori, on peut estimer normativement que le dirigeant devrait en priorité se consacrer à des activités telles que la cueillette d'informations sur le marché (Marchesnay, 1988, dans ce numéro), la prise de décisions de stratégie commerciale (choix des produits et des marchés, décisions du plan de marchéage - «marketing mix»); ce qui implique une certaine inclination, prédisposition de sa part dans la mesure où il se trouve généralement en situation de suroccupation de temps d'activité. Il n'est de secret pour personne que la littérature laisse entendre que les petites entreprises sont moins bien «gérées» que les grandes, que le chef d'entreprise planifie moins et se désintéresse des décisions stratégiques et par conséquent de l'activité de mercatique (Bourantas et Marchesnay, 1989).

- Le propriétaire-dirigeant est une... personne, et l'expression de ses aspirations personnelles a des conséquences décisives sur le choix de ses buts comme de son style de commandement. De nombreuses classifications ont été faites des entrepreneursindividuels, la plus connue étant celle de l'«entrepreneur-artisan» et de l'«entrepreneur-opportuniste». Lorrain et Dussault (1988) font apparaître que les entrepreneurs opportunistes, davantage ouverts sur l'environnement, accordent une place significative au marketing ( $25 \%$ d'un échantillon de 29 entrepreneurs) et que les entrepreneurs artisans y sont nettement moins sensibles (13\% d'un échantillon de 41 entrepreneurs). Bien plus, c'est pour le marketing que la valeur $U$ du test de Mann-Witney est la plus significativement différente. 
Pour notre part, nous avons distingué deux types d'entrepreneurs (Marchesnay, 1986) :

- L'entrepreneur «PIC», qui recherche surtout la pérennité de son affaire, veut garder son indépendance patrimoniale, et n'accepte la croissance qu'à ce prix, sous réserve de ne pas dépasser un seuil.

- L'entrepreneur "CAP», qui cherche la croissance de son marché - quitte à externaliser certaines activités ou fonctions pour ne pas développer l'organisation -, qui veut conserver l'autonomie de direction, et qui n'accepte de pérenniser l'activité que sous réserve de développer les aspirations précédentes.

Le «CAP» semble donc plus enclin à s'intéresser aux problèmes de marché et le «PIC», aux problèmes internes (production et personnel). Leur vision du marketing risque d'être différente. Mais, dans la réalité concrète, les types d'entrepreneurs perçus sont souvent mixtes, ne serait-ce que parce que les entrepreneurs n'ont pas toujours une conscience claire de leurs aspirations, ou de leur côté contradictoire.

L'une des conséquences du rôle primordial de l'entrepreneur est de faire glisser au second plan le rôle de l'organisation de la petite entreprise. Toutefois, on doit constater que plus l'entreprise grandit, plus elle subit des effets de seuil qui se manifestent notamment par la création d'une hiérarchie, et impliquent la création de services et l'embauche de personnel (Mahé de Boislandelle, 1988) : on est alors aux limites de la moyenne entreprise. Plüs la petite entreprise gagne en maturité et suit le développement de son marché, plus elle se rapproche de cette situation, et plus se posent des problèmes liés davantage à la gestion commerciale (service commercial, embauche d'une force de vente) qu'à la stratégie commerciale (choix des produitsmarchés.

\subsection{Un environnement très influent}

On a pu dire que si la grande entreprise modèle son environnement, la moyenne entreprise négocie avec celui-ci, tandis que la petite entreprise s'y adapte. L'environnement comprend trois niveaux distincts: les «forces du marché» (l'industrie au sens de Porter), la filière de relations avec les clients et les fournisseurs et enfin, la concurrence. Ces trois niveaux déterminent une situation propreà chaque entreprise et la prédispose à une activité commerciale plus ou moins intense.

- L'environnement industriel, par sa nature plus ou moins complexe, plus ou moins turbulente, plus ou moins accessible, rend la petite entreprise plus ou moins vulnérable à ses évolutions. Le degré plus ou moins élevé de vulnérabilité est donc fonction (Marchesnay, 1986) :

- du degré de complexité des technologies de production et de distribution repéré par des indications telles que : le nombre des relations entre les opérations, le degré de qualification, la spécialisation des appareils, la sophistication des systèmes d'information, etc.; 
- du degré d'accessibilité des systèmes de production et de distribution, repéré par : le faible coût d'entrée (au sens large) et la vitesse d'apprentissage de la technologie et du marché;

- du degré de turbulence, repéré par la fréquence et l'ampleur des changements dans les technologies et dans les goûts des clients.

La petite entreprise apparaît plus particulièrement sensible, potentiellement, à son environnement. Elle sera fortement vulnérable dans un environnement complexe, accessible et turbulent. Elle sera moins vulnérable dans un environnement peu complexe, peuaccessible, et peu turbulent:c'est plaider pour une stratégie commerciale axée sur les créneaux. Par ailleurs, afin de lutter et de s'immuniser partiellement contre la vulnérabilité, la petite entreprise devra mettre en place une triple capacité : - une capacité de mâtrise de la complexité, par une formation et une information adaptée : veille technologique et commerciale (Marchesnay, 1986);

- une capacité de réduction de l' accessibilité, ou tout au moins, de surveillance des nouveaux entrants, ou des nouveaux produits de substitution, impliquant là encore une activité de veille;

- une capacité de pilotage impliquant une adaptation rapide face aux turbulences, toujours grâce à l'activité de veille.

Il serait intéressant de connaître les modalités de prise en charge de cette dernière dans les petites et toutes petites entreprises. On peut penser qu'elle est assumée par le propriétaire-dirigeant, mais d'une façon plus pragmatique et occasionnelle que systématique, plus réactive que proactive.

De même, on peut supposer que plus l'environnement est source de vulnérabilité, plus l'entrepreneur consacre une part importante de son temps à l'activité commerciale et de marché2.

- Mais il convient de coupler la variable «vulnérabilité» à la variable «dépendance». En effet, dans les relations avec les clients et les fournisseurs, la petite entreprise risque de se trouver en situation d'infériorité, à cause d'une forte dépendance vis-à-vis de l'un ou de plusieurs des protagonistes. Reprenant la littérature consacrée à la dépendance (Marchesnay, 1979), nous suggérons trois critères successifs pour repérer les zones de dépendance :

- repérer si, sur un flux, il n'y a qu'un seul partenaire (forte concentration) ou quelques-uns seulement;

- repérer si ce partenaire est irremplaçable, ou difficilement remplaçable (faible substituabilite);

- repérer, enfin, si cet échange est essentiel dans la stratégie de l'entreprise.

2 Boag et Dastmalchian (1985) définissent la vulnérabilité comme «le degré de dépendance d'une firme par rapport aux autres firmes, groupes ou individus». Les auteurs font à notre sens une confusion regrettable entre la vulnérabilité et la dépendance : on peut être vulnérable sans être dépendant, et réciproquement. Ils sont par ailleurs contraints (p. 39) d'ajouter l'incertitude (c'est-à-dire la turbulence) dans leur analyse. Notre grille apparaît à la fois plus compréhensible et plus compréhensive. 
Si nous répondons à ces trois éléments, l'entreprise est dite dépendante de son partenaire. Dans ces circonstances, on peut s'attendre à ce que le chef d'entreprise manifeste une activité plus intense en matière commerciale, afin de trouver d'autres partenaires. Cette activité sera différente selon que la dépendance se situe en amont ou en aval. D'un autre côté, on peut imaginer que la petite entreprise est fortement dépendante en amont et/ou en aval : en ce cas, sa stratégie commerciale se trouve contrainte au point que le chef d'entreprise peut littéralement s'en passer - avec tous les dangers que cela implique, puisque ce sont le ou les partenaires qui s'en chargeront : à ce moment, des transferts de surplus sont à craindre (Mahé, 1984).

\section{concurrentiel.}

- Enfin, la petite entreprise doit tenir compte de son environnement

La coutume veut que cet environnement soit particulièrement agressif, et que l'entreprise en soit réduite à une lutte à couteaux tirés, au travers de la recherche du prix minimum. En fait, le «marché» a souvent une allure clanique : une logique institutionnelle s'instaure au sein de la profession; on a davantage affaire à des «collègues» ou «confrères» qu'à des adversaires. L'entreprise pourra se localiser dans un «créneau» complémentaire.

Une autre idée reçue veut que ces petites entreprises aient un marché étroit, local. Bien des petites entreprises modernes ont un marché national, voire international, la localisation in situ pouvant davantage relever d'opportunités diverses (contraintes personnelles, aménités offertes, etc.) qu'à la présence du marché. Tel est le cas des entreprises spécialisées.

On peut penser que plus la concurrence est agressive, plus le marché est large (ce qui accroît la probabilité d'existence de concurrents), plus l'activité commerciale du dirigeant devrait être importante.

Au total, la petite entreprise est donc très influencée par son environnement, que ce soit en termes de vulnérabilité, de dépendance, ou d'agressivité. Pour se prémunir face à une telle situation, la petite entreprise va se localiser dans des activités qui la protègent relativement.

\subsection{Des activités spécialisées}

Dans l'imaginaire non scientifique, la petite entreprise serait vouée à subir une concurrence des prix, ce qui la rendrait non compétitive. Dans un article récent, déjà cité, J. Kinsey (1987) affirme que (p. 19) : «les observations suggèrent que plusieurs petites firmes vendent trop peu cher («under price»)». Une telle affirmation mérite d'être fortement nuancée. Comme le rappelle le même auteur, la littérature s'accorde pour prédire longue vie à la petite entreprise qui tient un «bon» produit, qui se localise dans un segment de marché et qui fournit un service spécifique. Bref, les petites entreprises ont intérêt à choisir une stratégie générique axée sur la «différenciation» et la spécialisation en s'attachant la clientèle, en la fidélisant par une interaction constante qui valorise le produit fourni par les services rendus autour 
de la vente. En conséquence, le prix n'est plus la variable essentielle, mais la «qualité» :

- «Qualité» de la relation avec les clients et les fournisseurs; ceux-ci participent, surtout dans les productions sur spécifications des clients, au processus de production et, comme dans les activités de service, agissent pratiquement comme un facteur de production. Cela implique un système d'interaction très élaboré (Marchesnay et Rudel, 1985), des contacts approfondis entre le propriétaire-dirigeant (et les membres del'entreprise) et les partenaires extérieurs. En ce sens, la nature de l'activité commerciale du propriétaire-dirigeant de petite entreprise est d'une nature très spéciale, et parfois difficilement sécable des autres relations d'affaires.

- "Qualité» de la prestation fournie aux clients : plus que dans la grande entreprise, qui médiatise sa prestation au travers d'un réseau de distribution, la petite entreprise évalue très rapidement le degré de satisfaction de la clientèle. Elle doit satisfaire un besoin (souvent exprimé lors de la spécification du produit par le client). Elle vend, bien souvent, un ensemble «produit-service», et la relation commerciale se trouve étroitement combinée à la relation de production d'un bien. En ce sens, on peut estimer que le dirigeant de petite entreprise devrait posséder un esprit commercial développé, mais que celui-ci va se trouver englobé dans des impératifs de production, surtout dans les activités destinées aux autres entreprises. Il en résulte que la spécialisation, le «créneau», va se définir davantage en fonction de considérations techniques que de raisons commerciales.

Au total, la nature de la petite entreprise ne va pas sans quelques paradoxes : de par sa situation, le rôle de l'activité commerciale et des décisions de marché devrait être déterminant. Mais on peut se demander si, dans le quotidien, d'autres types de décisions ne tendent pas à prendre le pas. C'est pourquoi l'observation concrète de quelques entreprises, représentatives de situations typées, s'avère nécessaire.

\section{$2 \quad$ Nécessité d'enquêtes exploratoires}

A ce stade de la recherche, il est difficile de se lancer dans une enquête approfondie : les liaisons entre les variables ne sont pas bien établies; on se heurte à l'extrême diversité des petites entreprises, en fonction notamment de la taille et du secteur d'activité. Enfin, l'enquête de terrain doit être privilégiée dans de telles recherches, car on réduit ainsi des biais très caractéristiques de l'enquête postale, biais relatifs à la perception par le chef d'entreprise de ses propres problèmes - sans être assuré de les éliminer totalement-, à moins d'adopter une démarche diagnostique plus longue et plus coûteuse en temps. 
J. Kinsey (1987) adopte une démarche similaire à la nôtre. L'auteur retient 50 entreprises, avec entretien d'une heure à une heure et demie : 42 entreprises dans le secteur des produits aux entreprises, 12 dans le secteur des produits aux particuliers, 5 dans le secteur des produits aux administrations. Toutefois, les entreprises vont de 5 à 500 personnes, alors que notre enquête ne retient que les entreprises de moins de 50 personnes. Mais nous évoquerons un certain nombre de conclusions et d'observations de l'étude de Kinsey.

L'étude que nous avons dirigée a porté au total sur 37 entreprises, en trois groupes distincts.

- La première enquête a concerné 10 toutes petites entreprises (moins de 10 salariés), jeunes (en phase de démarrage), de technologie de pointe (produits destinés aux entreprises, dans des secteurs nouveaux). Ces entreprises sont localisées dans la pépinière d'entreprises de Montpellier, soutenue par la Communauté Européenne, en tant que Centre d'Innovation. La région de Montpellier s'enorgueillit de posséder une technopole, avec quatre pôles technologiques (Ayral, 1988).

- La seconde enquête porte sur 12 entreprises de la région de Grenoble (Louis, 1989) : 7 sont des petites entreprises (moins de 50 salariés), 5 sont des toutes petites entreprises (moins de 10 salariés) dans les services et dans l'industrie. Ces entreprises sont situées dans la ZIRST, c'est-à-dire la technopole de Grenoble. Comme Montpellier, Grenoble est une ville dynamique, disposant de centres de recherche importants, mais c'est de surcroît une ville fortement industrielle, en particulier dans des activités de pointe, bénéficiant tout autant de la proximité de la Suisse que de celle de Lyon. Ces entreprises ont de 5 à 8 ans, c'est-à-dire qu'elles ont dépassé la phase de démarrage dans laquelle se trouvent encore les T.P.E. montpelliéraines étudiées.

- La troisième enquête porte sur 15 entreprises industrielles de la région de Nîmes (entre 20 et 30 salariés), ayant de 3 à 9 ans d'existence. La ville de Nîmes possède une tradition industrielle héritée du dix-neuvième siècle, mais dans des secteurs de la première génération industrielle (textile surtout, «petite mécanique»). La ville a donc connu une grave crise industrielle au cours des 20 dernières années, et la municipalité souhaite voir se développer de nouvelles entreprises, essentiellement industrielles, sachant que Montpellier, distant de 50 kilomètres seulement draine les activités de service et, sans doute, de pointe (Blanc, 1989).

Il est donc particulièrement intéressant de comparer ces trois groupes, afin de relever ce qui les différencie et ce qui les rassemble.

Le questionnaire, établi lors de la première enquête (Ayral, 1989), reprend les thèmes évoqués au début de cette contribution : identification de l'entreprise, puis du dirigeant, connaissance de l'environnement (les clients, les fournisseurs, les concurrents, les partenaires, attitude face au risque externe), problèmes commerciaux (détection, attitude, perception de la dépendance et de la vulnérabilité, nature des décisions commerciales), stratégie commerciale (externalisation éventuelle, perspectives d'avenir, contraintes et opportunités). 
A partir des rubriques de ce questionnaire, il est possible de formuler un certain nombre de réflexions. Répétons qu'il ne saurait s'agir d'une validation logico-statistique, mais bien plutôt d'un ensemble de considérations susceptibles d'étayer des voies de recherche dans l'édification d'une mercatique de la petite et toute petite entreprise qui nous fait cruellement défaut. Nous nous attacherons à mettre en valeur le rôle de l'entrepreneur, de l'environnement et de l'activité dans la mise en pratique de l'action commerciale de la petite entreprise.

\section{Profil du propriétaire-dirigeant et attitude vis-à-vis du marché}

Nous avons privilégié deux profils-types de propriétaires-dirigeants : le «PIC» et le «CAP» ou, approximativement, l'«artisan» et l'«opportuniste». Il convient, au préalable, de s'en tenir à des données factuelles : niveau et type de formation, degré d'expérience. D'ores et déjà, apparaît une très grande diversité.

Sur Montpellier, les dirigeants de ces jeunes T.P.E. se scindent en trois groupes :

- ceux qui sont formés dans le métier et expérimentés; ces créateurs ont dépassé la trentaine;

- ceux qui sont formés sur le métier, mais ont une expérience en dehors du métier : ils avoisinent la trentaine;

- ceux qui sont formés sur le métier, qui possèdent le savoir-faire, mais qui n'ont aucune expérience; il s'agit de jeunes créateurs, frais émoulus du système éducatif montpelliérain.

On pourrait s'attendre à ce que l'attitude commerciale soit différenciée nettement entre ces trois types d'entrepreneurs. La suite de l'enquête montre plutôt des attitudes communes.

En ce qui concerne Grenoble, l'enquêtrice fait apparaître une coupure nette dans l'échantillon :

- Les entrepreneurs sont formés et expérimentés dans le métier pour les activités industrielles.

- Pour les activités de services, il semble que les entrepreneurs aient changé de métier, ce qui correspond à une réorientation de carrière, au gré d'opportunités. Il semble que l'on ait affaire au type de «nouvel entrepreneur», mis en évidence par Sweeney, qui s'est développé dans les années 80-85 (Marchesnay, 1986) ce qui correspond assez bien à l'âge des entreprises ( 5 à 8 ans). En ce qui concerne Nîmes, les entrepreneurs sont plus âgés lors de la création (39 ans). Les 2/3 ont une formation supérieure et la moitié a une expérience dans le même métier, à titre de cadre pour l'essentiel. 
Au total, prédomine le profil du technicien ayant un savoir-faire dans une certaine activité. Mais ce profil a une très grande hétérogénéité, laquelle devrait s'éclairer à l'analyse des mobiles de la création.

Sur Montpellier, la création repose davantage sur la saisie d'une opportunité liée à la mise au point d'un produit ou d'un processus que sur la prise de conscience et, a fortiori, sur l'évaluation du marché. Cette attitude nous a été confirmée lors des entretiens du comité d'agrément de la pépinière, auquel nous participons. Comme le fait remarquer G. Hills (1985), le créateur ne fait pas d'étude de marché, se comporte de façon largement intuitive et surestime la demande «pour impressionner le bailleur de fonds». D'ores et déjà, nous pressentons une attitude peu propice à une approche mercatique.

Cette appréhension se trouve confortée par les observations sur Grenoble. La création est partie de l' «idée» d'un produit (bien ou service) industriel. Il est vrai que, dans un tissu industriel suffisamment dense (comme sur la ZIRST), l'idée trouve sa justification concrète dans une demande peu ou mal satisfaite de la part des entreprises : aussi bien souvent, et c'est un point capital, bien des créations de T.P.E. et P.E. sont-elles suscitées et encouragées par les futurs clients eux-mêmes (quitte à favoriser des essaimages), ce qui explique la nature très particulière des relations commerciales qui vont se nouer.

Ce sentiment est conforté par l'analyse des réponses sur Nîmes. Ici, le tissu industriel est distendu, et les clients seront bien souvent extérieurs. Les raisons de l'implantation reposent fortement sur des considérations personnelles (climat, liens familiaux) plutôt que de marché. L'idée de travailler «pour son propre compte» recueille un tiers des suffrages, ce qui est en relation avec l'âge plus prononcé des créateurs. La saisie d'une opportunité n'est reconnue que dans la moitié des cas. Faute de liens commerciaux suffisamment forts, la croissance du chiffre d'affaires apparaît primordiale.

Ainsi, une étude, aussi superficielle soit-elle, des propriétaires-dirigeants, nous éloigne de toute attitude normative et, a fortiori, moniste. On a plutôt affaire à des types de dirigeants assez marqués. Mais, en même temps, il semble bien que, de façon générale, la création soit avant tout saisie autour de l' «idée», c'est-à-dire d'un produit, d'un savoir-faire, et non d'un «marché», ce qui nous éloigne de la conception traditionnelle de l'esprit d'entreprise inculquée par l'idéologie libérale. Mais le rôle de l'environnement immédiat semble ici déterminant. 


\section{$4 \quad$ Nature de l'environnement et attitude mercatique}

Nous avons retenu, comme variables-clés, la vulnérabilité, la dépendance et l'agressivité dans les trois zones-clés de l'environnement spécifié (industrie, filière, concurrence). Les réponses foumies sont à ce sujet éclairantes.

Il convient de noter que les réponses constituent en fait des perceptions des chefs d'entreprise, sur des domaines ou des questions qui ne leur sont pas toujours très familiers.Aussi, peuvent-ilssurestimer lesrisques ou, au contraire, les opportunités.

Dans le cas des entreprises montpelliéraines, de jeunes T.P.E., la vulnérabilité technologique peutêtre considérée comme forte. En fait, ces entrepreneurs s'attachent le plus souvent à maîtriser la complexité en augmentant leur savoir-faire sur des produits ou des procédés qui ont largement justifié la création. Ils cherchent à réduire l'accessibilité en spécifiant davantage l'originalité de leur produit, en le différenciant par des services, en fidélisant la clientèle. Ils cherchent enfin à réduire les turbulences, en raccordant le produit-service à une filière de transformation. Ces points sont bien perçus par le dirigeant. En revanche, les aspects de vulnérabilité commerciale sont à peine esquissés. Le leitmotiv est : "C'est important, mais on n'a pas encore eu le temps d'y penser...». En conséquence, le jeune dirigeant se raccroche aux relations avec la clientèle, voire le client, lequel pourra être à la base de la création. Ce faisant, cette clientèle fidélisée contribue à réduire la vulnérabilité; mais elle augmente la dépendance de la petite entreprise. Le risque est alors que le dirigeant, enferré dans les problèmes de maîtrise technologique, néglige de rechercher d'autres clients, d'élargir son marché. Mais, en règle générale, les chefs d'entreprise sont conscients de ce problème, même s'ils n'y portent pas immédiatement remède. Mais ceci impliquerait d'entreprendre une étude du marché qui, n'ayant pas été faite au départ, ou de façon superficielle, s'avère coûteuse à mettre en oeuvre (en temps comme en argent). En revanche, la dépendance à l'égard des fournisseurs est nettement moins ressentie : $s$ 'ils sont souvent concentrés, les chefs d'entreprise pensent qu'ils sont substituables. Mais, en fait, ils contribuent souvent à la maîtrise de la complexité technologique.

L'étude des entreprises grenobloises est éclairante: plus «vieilles», elles ont dépassé le stade du démarrage. En conséquence, la complexité technologique est maîtrisée et la vulnérabilité technologique apparemment réduite. Le problème de la vulnérabilité commerciale et de la dépendance à l'égard de gros clients apparaît plus critique, et les entrepreneurs déclarent avoir clairement conscience du problème. Toutefois, ils s'attachent parallèlement à fidéliser la clientèle acquise en adaptant les produits-services aux besoins des clients. On peut estimer qu'une certaine sensibilisation commerciale est dès lors opérée, d'autant plus que les entreprises cherchent à élargir le champ des partenaires avec lesquelles elles sont en relation étroite (fournisseurs, bailleurs de fonds). La politique de fidélisation des fournisseurs est accentuée (ce qui limite à l'évidence les coûts de transaction). Ceci n'est rendu possible que par la densité du tissu industriel sur la région grenobloise. Il est permis, 
par exemple, de s'interroger sur la possibilité, pour les entreprises montpelliéraines, d'accéder à ce stade, compte tenu de la faible densité des partenaires dans la région.

Si l'on considère, en effet, les entreprises nîmoises, on constate qu'elles sont contraintes de s'ouvrir sur des marchés non régionaux : les $2 / 3$ des entreprises contactées ont un marché international. En conséquence, elles se perçoivent comme plus vulnérables. Le nombre de clients est plus important (entre 300 et 600) et, pour la moitié, elles doivent procéder à un démarchage systématique tout en reconnaissant, pour un tiers d'entre elles, le rôle de la demande spontanée qui a pu être à l'origine de lacréation. Cette forte vulnérabilité ressentie (et peut-être surestimée) s'accompagne d'une faible dépendance, mais aussi d'une assez bonne fidélisation (les clients sont jugés «réguliers» à $90 \%$ ), ce qui implique une bonne flexibilité technique afin de répondre rapidement aux spécifications des clients. Mais l'accessibilité est ressentie à $50 \%$ comme forte; et, surtout, la turbulence commerciale comme l'agressivité de la concurrence sont évaluées comme fortes pour 80 à $90 \%$ des entrepreneurs; ce qui aurait dû susciter une politique commerciale active.

Au total, ces quelques observations suffisent, pensons-nous, à faire apparaître combien les situations des P.E. et T.P.E. sont diversifiées. Mais on doit retenir combien le rôle du client et de sa fidélisation est important, voire déterminant pour la survie. De même, le fournisseur apparaît nettement comme un partenaire ou permet de résoudre les problèmes techniques. Mais le sentiment général prévaut qu'une priorité est accordée à ces derniers, relativement aux problèmes commerciaux et de marché. Les observations suivantes confirment largement cette intuition.

\section{$5 \quad$ Nature de l'activité et compétitivité commerciale}

L'opinion selon laquelle le propriétaire-dirigeant de petite entreprise ne se préoccupe pas des problèmes commerciaux mérite d'être soigneusement nuancée. La difficulté vient de ce que la relation avec le client est largement issue des problèmes techniques qu'il convient d'élucider et de résoudre ensemble, plus que d'une appréhension opportuniste du marché; en d'autres termes, les besoins, envisagés sous leur angle technique, sont bien appréhendés : mais, faute d'un état d'esprit mercatique, il n'en découle pas de réelle stratégie commerciale.

Les entreprises montpelliéraines se fondent avant tout sur leur capacité d'innovation : celle-ci apparaît comme une réponse à l'extrême turbulence de leur environnement. Cette stratégie implique de se tenir étroitement au courant des besoins de la clientèle, d'autant plus que la concurrence directe est difficile à maîtriser. Cela entraîne sur le plan de marchéage (ou «marketing mix») plusieurs conséquences, qui feraient frémir un spécialiste de mercatique appliquée à la moyenne ou grande entreprise : 
- La distribution se fait directement auprès du client. Toutefois, passée la phase de démarrage, le souci d'éviter la dépendance conduit à rechercher l'élargissement du marché. Pour ces jeunes entreprises, le besoin d'études de marché, et, surtout, d'une force de vente, commence à se faire sentir sans qu'on sache très bien comment le résoudre : il s'agit en fait d'une décision plus proactive que réactive, et les jeunes dirigeants, souvent inexpérimentés, n'y sont pas habitués(Bourantas et Marchesnay, 1989; Grognier, 1988; Alpander, Carter et Forsgren, 1988). Bref, l'absence d'une planification de stratégie commerciale se fait sentir à cette phase cruciale : la décision adaptative, réactive, intuitive, ne suffit plus.

- Il n'y a pas de réelle politique de prix. Le flou s'explique ici par le caractère innovant et la volonté de se différencier par rapport à une éventuelle concurrence. Par ailleurs, ces produits ne font pas l'objet d'un échange sur un marché analogue à celui des biens de consommation : le prix est le résultat d'une négociation avec le client, qui impose certaines spécifications, sous la réserve d'accepter une certaine «élasticité» dans le prix de cession. Il est toutefois difficile de rationaliser en termes de «cost-plus», car il semble bien que peu de dirigeants aient une connaissance exacte du coût de revient complet de chaque prestation.

- On conçoit aisément que, dans ces conditions, la politique de promotion, de publicité et de communication soit quasi inexistante. A l'instar des autres entreprises que nous allons voir, celles-ci se contentent largement d'une présence dans les salons, foires et expositions.

Cette attitude peut apparaître d'autant plus étonnante que ces entreprises sont localisées dans une «pépinière», qui les sensibilise à la mercatique et à l'action commerciale et qui met à leur disposition des conseils en marketing. Toutefois, la nature de l'activité (jeune, destinée à des industriels, très positionnée) peut expliquer en partie cette méconnaissance, sinon la justifier.

Si l'on étudie les entreprises grenobloises, on constate que la politique commerciale est assumée par le dirigeant dans la quasi-totalité des cas. Les traits les plus remarquables sont à nos yeux les suivants :

- La plupart du temps, il n'y a pas de service commercial. Lorsque ce service existe, les employés commerciaux dépendent directement du dirigeant; en définitive, n'est-ce pas montrer l'importance stratégique de cette activité ? Mais, d'un autre côté, les chefs d'entreprise souhaitent recruter des technicocommerciaux, du moins dans les activités industrielles. Dans les services, les entrepreneurs souhaitent conserver la maîtrise du contact avec le client. Une des raisons fréquemment invoquée est le risque de voir les vendeurs constituer leur propre entreprise avec la clientèle, les garanties contractuelles s'avérant en l'espèce extrêmement fragiles.

- Il n'y a pas d'études de marché. Seulement la moitié des entreprises avaient fait une étude de marché avant le démarrage (encore eut-il fallu la voir...). Il n'y a pas de politique promotionnelle : on se contente d'un «marketing direct», sous forme de plaquettes, de publipostage («mailing»), pour un quart des entreprises. Mais les entrepreneurs se plaignent de l'efficacité douteuse de ces procédés 
promotionnelsetpréferent s'en tenir à la présence aux foires, expositions, salons. Le parrainage («sponsoring») est peu usité, sauf pour des raisons personnelles au dirigeant.

- Là encore, la pratique commerciale passe par l'interaction avec le client, voire avec l'ensemble des partenaires («concurrents» fabriquant des produits voisins ou complémentaires), car le tissu industriel est dense. En conséquence, la compétitivité ne repose pas sur le prix, mais sur la «qualité». Ce terme est souvent invoqué, mais reste mal défini. Il semble correspondre à la satisfaction du client, laquelle, par le bouche à oreille, permettra de trouver de nouveaux clients. La "qualité» consiste également à trouver des produits adaptésaux besoins exprimés par les clients. La demande spontanée est ici déterminante : c'est le client qui, de fait, imprime sa marque à la politique de produit, bien que deux tiers des entreprises industrielles souhaitent recruter dans un avenir proche des technicocommerciaux.

Ainsi se trouve confirmé le sentiment d'une pratique commerciale, plus que d'une véritable politique commerciale.

Ce sentiment est conforté par les P.E. nîmoises.

- Là encore, c'est le dirigeant qui assume l'activité commerciale, seul dans la moitié des cas, avec un ou plusieurs collaborateurs dans un quart des cas. Davantage conditionnées par le marché non régional, comme on l'a dit précédemment, ces entreprises ont un service commercial en quasi-totalité. Mais la distribution (contact client) est le fait du dirigeant dans les deux tiers des cas, de sorte que ce service s'occupe avant tout de gestion, plus que de négociation commerciale.

- Cette absence d'esprit mercatique est soulignée par le fait que les deux tiers n'ont pas fait d'étude de marché à la création, que les trois quarts n'en ressentent pas le besoin, et n'éprouvent pas également le besoin de conseillers extérieurs (pour l'autre quart, le conseil en marketing se réduit à la création d'un logo ou d'un slogan, à une opération de publipostage).

- De même, le budget de publicité reste minime (et d'ailleurs mal connu par bien des enquêtés) et se réduità quelques opérations simples: inscription sur les pages jaunes, plaquettes pour les foires et expositions, publicités dans les revues professionnelles ou municipales...

- Le prix, là également, n'est pas invoqué comme une arme essentielle, alors que la majorité des chefs d'entreprise ont invoqué l'agressivité de la concurrence... Plutôt, on fait référence à la «qualité», chez $70 \%$ des entreprises : cet argument peut, au demeurant, constituer un moyen de compenser psychologiquement l'absence de stratégie commerciale... Bref, la notoriété est le fruit de la satisfaction du client, qui se saura par le «bouche à oreille» («word of mouth», dit Kinsey).

- Mais, en même temps, les dirigeants ressentent un fort besoin de technicocommerciaux, même si, parallèlement, le rôle prépondérant est attribué au client 
et au contact direct avec le client. La moitié souhaitent recruter des vendeurs, $20 \%$ monter un réseau de distribution, mais $1 / 3$ ne perçoivent pas le sens de ce développement... La quasi-totalité voudrait se diversifier, trouver de nouveaux clients, de nouveaux marchés, de nouveaux produits. Mais la démarche apparaît nettement plus incrémentale que planificatrice; ce qui confirme l'observation de Kinsey selon qui 40 entreprises sur les 50 enquêtées n'ont pas de planification.

Au total, le sentiment prévaut que ces entreprises de petite taille se trouvent confrontées à un dilemme :

- D'un côté, le maintien de leur compétitivité se trouve lié au développement de leur marché et implique le passage à une organisation différenciée, hiérarchisée, avec notamment un service commercial étoffé et une politique commerciale élaborée - impliquant un surcroît de capitaux, sans doute extérieurs.

- D'un autre côté, ces entrepreneurs, plutôt de profil «PIC», souhaitent préserver leur autonomie de décision et leur indépendance financière, tout en conservant une organisation de type intégratif fortement adhocratique... sous la réserve que le propriétaire-dirigeant prenne seul les décisions importantes! Ainsi que le note Kinsey, que nous rejoignons, «la plupart des répondants ont le désir de demeurer relativement petits».

\section{Conclusion}

En conclusion, un certain nombre de traits communs semble se dessiner entre les P.E. et les T.P.E.; mais également, des différenciations se font jour, qui devraient déboucher sur des pistes de recherche.

En premier lieu, les observations de terrain rejoignent les idées reçues sur la petite entreprise :

- l'importance du rôle du dirigeant dans l'élaboration et la mise en oeuvre de la politique commerciale - comme pour les autres décisions;

- l'importance de la nature de l'industrie et du type d'activité : maturité, sophistication technologique, poids des services

- l'importance des problèmes de vulnérabilité et de dépendance, qui doivent être dissociés;

- l'importance de la délimitation de l'activité dans des frontières telles qu'elle satisfasse un besoin précis exprimé directement par le client;

- l'importance, enfin, du client. La petite entreprise a une clientèle, pas un marché. C'est la clientèle qui délimite ce marché. La concurrence est mal connue et la clientèle se manifeste souvent spontanément, du fait de la «qualité», de la notoriété supposée de l'entreprise, sans que les causes en soient systématiquement inventoriées.

La conséquence majeure est la faillite du «plan de marchéage» (marketing mix) dans la petite entreprise. Mais il faut se garder de tout jugement normatif : en 
particulier, la petite entreprise a-t-elle besoin de publicité, de politique promotionnelle ? A-t-elle besoin d'une organisation commerciale très structurée qui lui ferait perdre une partie de sa flexibilité ? Il semble que le problème ne se pose surtout que lorsque l'entreprise veut passer à une dimension supérieure de son marché, soit parce qu'elle y est contrainte, soit parce que son ou ses dirigeants le souhaitent. A ce moment, l'absence d'une stratégie mercatique se fait cruellement sentir et les atouts de la petite taille se muent en handicap. entreprises :

En second lieu, des différences sont néanmoins perceptibles entre ces

- Le profil du dirigeant devrait jouer un rôle important dans l'attitude face à la décision commerciale. La plupart sont apparus «technocentrés»: il convient de se demander s'il s'agit d'un hasard (le choix des entreprises dans des zones déterminées) ou d'une nécessité (bien maîtriser le métier). Il est remarquable de constater que peu ont su définir leur mission, opposée à leur métier. L'avènement d'une nouvelle génération d'entrepreneurs, davantage rompue aux méthodes modernes de gestion, va-t-elle modifier cette vision?

- Les attitudes devraient se différencier en fonction de la nature de l'activité, et de l'environnement. Il serait intéressant d'approfondir le rôle de la densité du tissu productif régional sur le comportement mercatique de l'entreprise. Les observations que nous avons faites sur ce sujet ne laissent pas d'être superficielles... De même, il conviendrait de mieux repérer, sans doute par des études cliniques, les éléments de vulnérabilité, de dépendance et d'agressivité effectifs, et de les comparer à ce qui est perçu par le propriétaire-dirigeant. Il serait également intéressant d'inventorier son processus de prise de décision, son attitude face au risque, etc.

Sur le plan de l'aide à la gestion de ces petites et toutes petites entreprises, la nécessité d'une démarche mercatique appropriée se fait sentir. Il ne suffit pas de s'offusquer de l'absence d'une démarche conforme aux enseignements des manuels, mais il faut encore se demander pourquoi la démarche est différente, et, finalement, si elle n'est pas largement adaptée aux besoins des entreprises de petite dimension, qui ne souhaitent pas dépasser un certain seuil. Si tel était le cas, cela soulèverait d'importantes questions sur la formation du dirigeant de toute petite entreprise, comme sur les activités de conseil à ces entreprises.

\section{BIBLIOGRAPHIE}

Alpander, G., Carter, K.D. et Forsgren, R. (1989), «Successful decision-making in the formative years of small business», W.P. University of Maine at Orono, 30 pages.

Ayral, S., (1988), «Le comportement commercial du dirigeant de petite entreprise : le cas de P.E. "high tech"», Rapport ORCES/ERFI-Montpellier, 165 pages.

Blanc, A., (1989), «Le comportement commercial du dirigeant de P.E. : le cas de P.E. nîmoises», mémoire en économie d'entreprise, Université de Montpellier, mai, 50 pages. 
Boag, D.A. et Dastmalchian, A., (1985), «Market vulnerability and the design and management of the marketing function in small firms», Journal of Small Business Management, janvier, pp. 38-46.

Bourantas, D. et Marchesnay, M., (1989), «Le processus de prise de décision stratégique des dirigeants de petite entreprise, Cahiers de recherche, Montpellier, mai.

Fallery, B., (1983), «Le système d'information du dirigeant de PME», thèse de doctorat, Université de Montpellier, 250 pages.

Faure, R., Marchesnay, M. et Mathie, B., (19..), «L'ombre des grands», Revue Française de Gestion, n 22, pp. 108-114.

Grognier, F., (1988), «Study of strategic decision-making behavior of small entreprises' owner managers», mémoire de doctorat, Université de Montpellier.

Hills, G., (1985), «Market analysis in the business plan : ventures capitalists' perceptions», Journal of Small Business Management, janvier, pp. 38-46.

Kinsey, J., (1987), «Marketing and small firms in Scotland», Journal of Small Business Management, vol. 25, $\mathrm{n}^{\circ}$ 2, avril, pp. 18-25.

Julien, P.A., et Marchesnay, M., (éd.) (1988), La petite entreprise.Principes d' économie et de gestion, Vuibert, Paris, 288 pages.

Julien, P.A., Chicha, J. et Joyal, A., (éd.), La PME dans un monde en mutation, Presses de l'Université du Québec, Québec, 445 pages.

Lorrain, J.et Dussault, L., (1988), «Les entrepreneurs artisans et opportunistes: une comparaison de leur comportement de gestion», Revue Internationale $P M E$, vol. $1, \mathrm{n}^{\circ} 2, \mathrm{pp} .157-176$.

Louis, C., (1989), «Le comportement commercial du dirigeant de petite entreprise : le cas de P.E. grenobloises», mémoire d'économie d'entreprise, Université de Montpellier, mai, 50 pages.

Mahe, H., (1984), «Un outil de diagnostic stratégique : la grille dépendance/technologie/ avantage», Economie et Sociétés. Sciences de Gestion, n 4, pp. 5-32.

Mahe de Boislandelle, H., (1988), Gestion des ressources humaines dans les P.M.E., Economica, Paris, 322 pages.

Marchesnay, M., (1979), «La dépendance des firmes individuelles : un essai d'analyse et d'application dans leurs relations d'échange avec les groupes", Economie et Sociétés. Sciences de Gestion, n 1, pp. 697-736.

Marchesnay, M. et Rudel, S., (1985), «La gestion du risque dans les T.P.E. Faits et théorie», Economie et Sociétés. Sciences de Gestion, no 6, pp. 43-76.

Marchesnay, M., (1986), La stratégie. Du diagnostic à la décision industrielle, Chotard, Paris, 248 pages.

Marchesnay, M., (1988), «De la veille technologique au pilotage stratégique», Revue Internationale $P M E$, vol. $1, \mathrm{n}^{\circ} 3$.

Marchesnay, M., (1988), «PME : quelle “techno-logique”?», Revue Internationale PME, vol. $1, n^{\circ} 2$.

Mintzberg, H., (1984), Le manager au quotidien, Editions d'organisation, Paris et Editions d'ARC, Montréal, 220 pages.

Naro, G., (1989), «Facteurs et comportements d'embauche dans les PME», thèse de doctorat, Université de Montpellier, 437 pages + annexes.

Saporta, B., (1986), Stratégies pour la PME, Entreprendre, Montchrestien, Paris. 